\title{
Research on Wireless Monitoring Technology of Smart Car
}

\author{
Zhang Lizhen ${ }^{\mathrm{a}, *}$, Chen Yulin, Zhang Sheng \\ Southeast University Chengxian College, Nanjing, Jiangsu 210088, China \\ azlz@cxxy.seu.edu.cn
}

Keywords: Smart Car, MCU, WIFI, Bluetooth

\begin{abstract}
This paper designs a smart car with two functions: automatic control and manual control. Under the mode of automatic control, the smart car can move on its own track. Under the mode of manual control, we can use the upper computer PC and mobile phone APP to control the free movement of the smart car. The smart car designed in this paper can achieve seamless switching between automatic control and manual control. It can not only avoid the uncertainty of the car's automatic movement, but also avoid the low efficiency of manual remote control, so as to maximize the intelligence of the car.
\end{abstract}

\section{Introduction}

The smart car, also known as the wheeled robot, is a comprehensive system which integrates the functions of environment perception, planning and decision-making and automatic driving. It is a typical high-tech complex with the technology of computer, sensing, information, communication, navigation, artificial intelligence and automatic control [1][2]. It has been widely used in the field of aerospace, ocean exploration, industrial and agricultural production, military field, social life and so on. It has a broad application and development prospects.

Wireless sensor network (wireless sensor network, WSN) is a flourishing remote monitoring technology, which is widely used in military reconnaissance, target tracking, environmental monitoring, medical and health, industrial control and business and other fields [3][4][5]. Therefore, a smart car based on wireless monitoring technology is designed, which takes the intelligent car as the mobile platform, and establishes wireless connection through the WIFI network environment. It can realize the use of PC and mobile APP to control the movement of the smart car, and the seamless handover between the automatic control mode and the manual mode, so as to maximize the car intelligence.

\section{System schematic}

The smart car designed in this paper has two functions: autonomous control and manual control. The smart car uses the MSP430F149 of TI company as the main control. Under the mode of autonomous tracking, it mainly uses gray sensor to detect track. Under the mode of manual control, the mobile APP and PC client are used to control the direction and speed of the smart car at the same time. the wireless connection between the mobile phone APP or the computer PC client and the control board of the car through the WIFI. Bluetooth is used to establish wireless connection between control board and communication driven steering gear. The system schematic diagram of the smart car is shown in Figure 1, and the practicality picture of the smart car is shown in Figure 2.

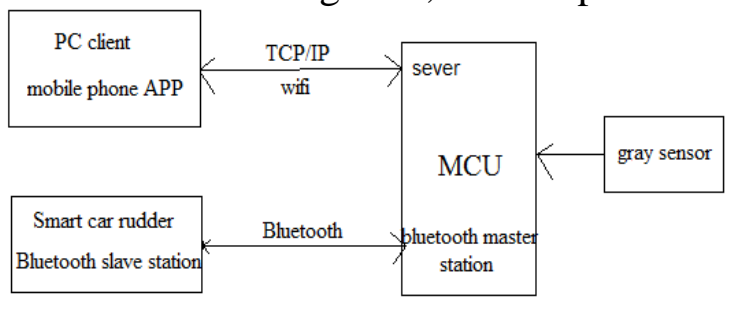

Figure 1 System schematic diagram

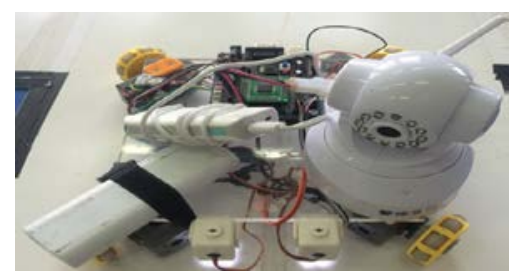

Figure 2 Practicality picture 


\section{Hardware Design}

The intelligent car system designed in this paper mainly includes smart car body, main controller, wireless communication Bluetooth module and wireless communication WIFI module. The main hardware parts are designed as follows:

\subsection{Design of the smart car body}

The body structure of the smart car is shown in Figure 3. The body structure of the smart car is driven by 90-degree omnidirectional wheel. The CDS5516 rudder is configured in a parallel way in four corners of the square floor, and the size is symmetrical, the positioning accuracy is $1 \mathrm{~mm}$, and the length and width of the car's floor is $200 \mathrm{~mm}$. Each of the four corners has two fixed holes for fixing the mounting base of the steering gear. The axis of the rudder should be symmetrical and square.

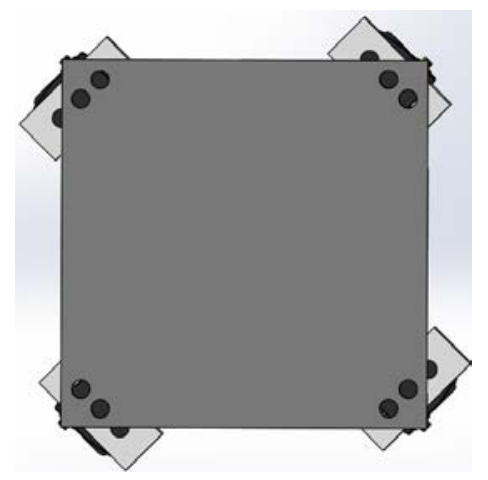

Figure 3 The body structure of the smart car

\subsection{Design of wireless Bluetooth module}

The control motherboard MSP430F149 MCU of the system mainly controls the movement of the smart car through Bluetooth. The wireless communication Bluetooth module used in this paper is shown in Figure 4. A schematic diagram of the connection between the Bluetooth module and the control motherboard is shown as shown in Figure 5. The RXD pin of the Bluetooth module is connected to the TXD pin of the single chip microcomputer, and the TXD pin of the Bluetooth module connects to the RXD pin of the single chip microcomputer. The transmission port of Bluetooth is connected to the SIG signal port of the steering gear. When the master sends data through Bluetooth, the Bluetooth receives and sends it to the steering gear.

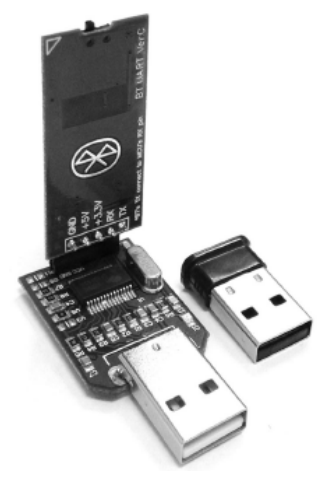

Figure 4 Bluetooth module

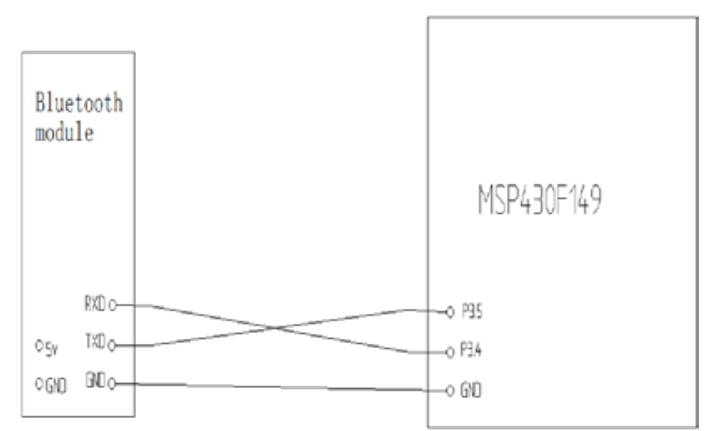

Figure 5 Schematic diagram of the connection

\subsection{Design of wireless WIFI module}

In this system, the WIFI module uses ESP8266. Its function and anti-interference ability are very strong. It can be used with the configuration software, and it can be used to solve the network scheme completely. The schematic diagram of the connection between the ESP8266 module and the master microcontroller MSP430FL49 is shown in Figure 6. 


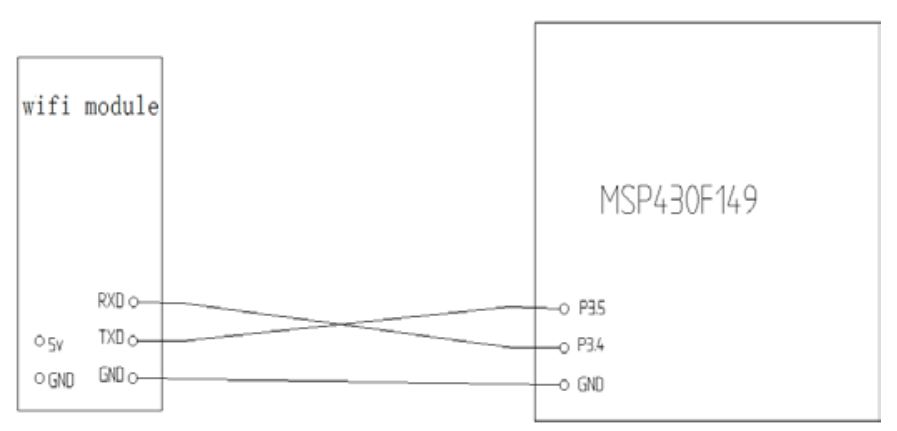

Figure 6 Schematic diagram of the connection

In the above figure, the RXD pin of the WIFI module is connected to the TXD pin of the main controller, and the TXD pin of the WIFI module is connected with the RXD pin of the main controller.

\section{Development of system software}

The software development of the system mainly includes the development of mobile phone APP control interface, the development of PC client control interface and the development of single chip computer program. The specific design is as follows:

\subsection{Design of mobile phone APP control interface}

The APP control interface of the system is mainly developed by Yi Android E4A programming tool. E4A is a programming tool based on the Google Simple language. It has a similar development environment as Chinese language. Its syntax prompts are not only powerful but intelligent, which are designed to easily write Android applications using basic syntax. It has the characteristics of easy to develop, easy to use and so on.

The APP control interface of the smart car is shown in Figure 7. The control interface is mainly composed of three control parts: the first part is the direction control part, including the move buttons in every direction; The second part is speed control part, including high, medium, low speed and stop button; The third part is the switch button between automatic mode and manual mode.

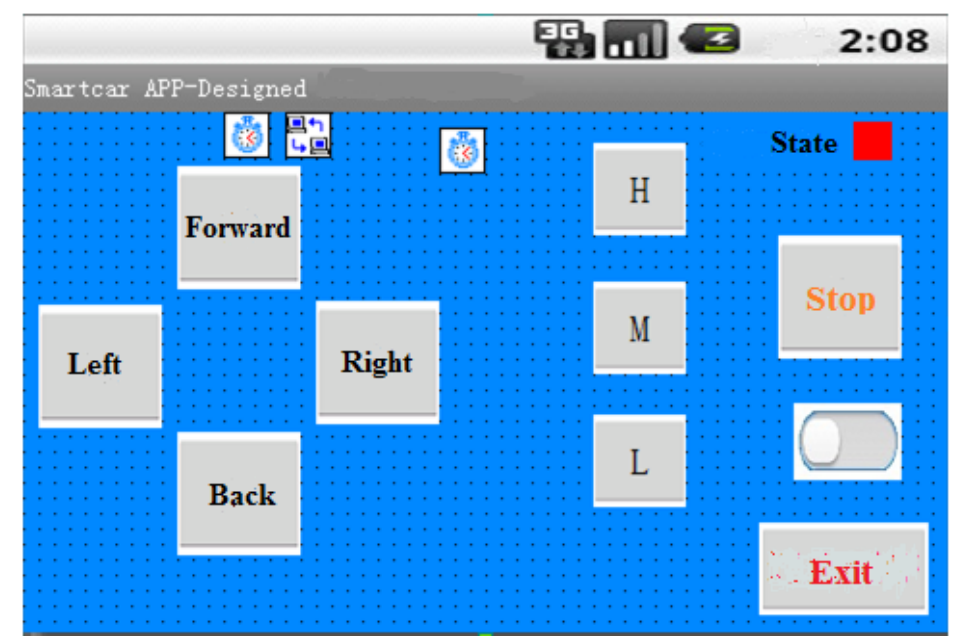

Figure7 APP control interface for smart car

\subsection{Design of $P C$ client control interface}

The PC client interface of smart car is shown in Figure 8. It contains four main parts, namely, the main interface, the test interface, the control interface and the parameter setting interface. The main interface is mainly used to remind developers of relevant information. The test interface is used to test whether the communication is successful, including the IP address, port number, connection button, disconnect button, send data button, and exit program button. The control interface is used to control the movement direction and speed of the smart car and switch automatic control and 
manual control, as shown in Figure 8. The parameter setting interface is mainly used to set the specific value of speed in high and low grade shown in the control interface.

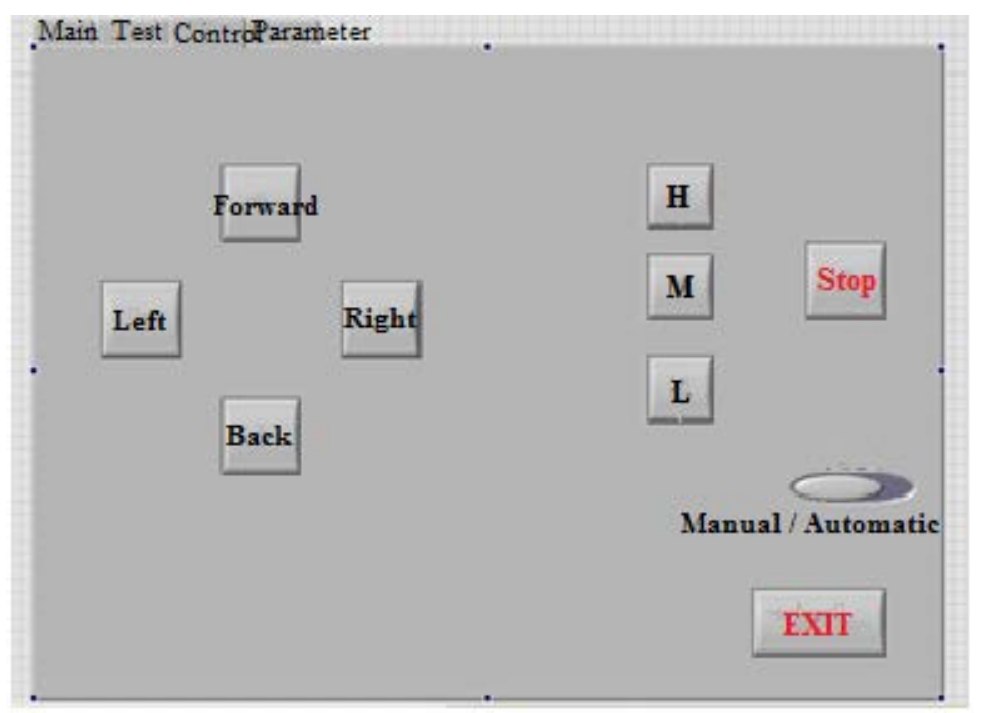

Figure 8 The control interface of PC client

\subsection{Program design of single chip microcomputer}

The MCU program mainly includes two parts: automatic mode program and manual mode program. When the program is initialized, two serial UART0 and UART1 corresponding registers are configured, and then the reception interrupt of the UART0 is opened to receive the data sent from the mobile phone APP and PC client. When the data is received, the receiving interruption is closed and the received data is judged and processed to distinguish the direction and speed information. Finally, the command is sent to the steering engine to control its steering through the serial port UART1. The schematic diagram of the master control program is shown in Figure 9.

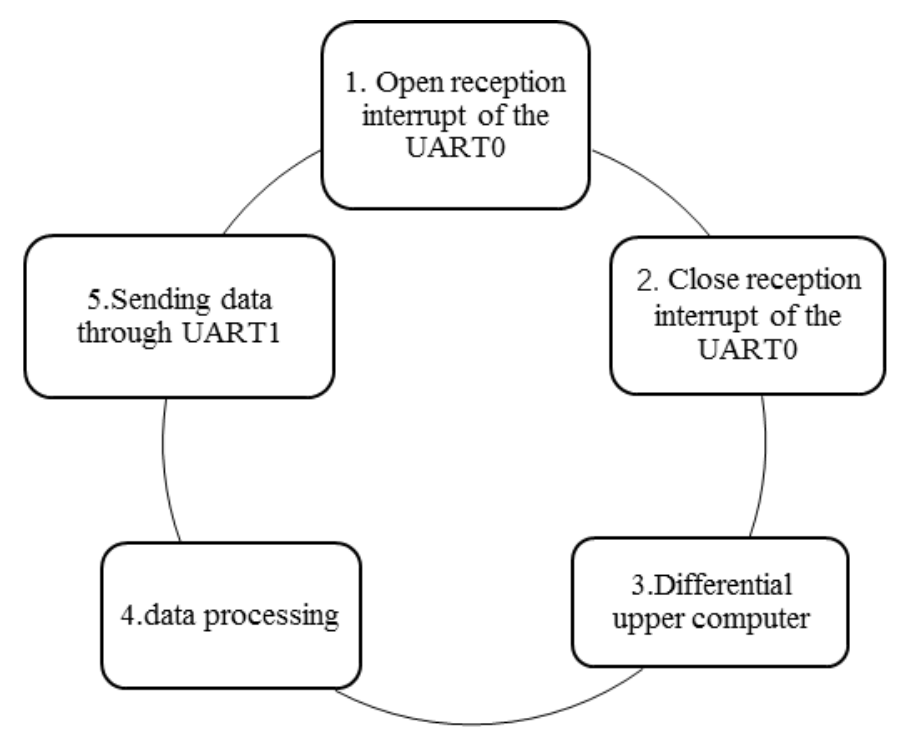

Figure 9 Design schematic diagram of the MCU program

\section{Conclusion}

In this paper, a smart car with two functions of automatic control and manual control is designed. The test shows that the smart car can detect and follow track well in the automatic control mode. Under the manual control mode, the mobile APP and PC client can control the moving direction and speed of the smart car at the same time. The smart car can be free switching in automatic control and manual control mode, which avoids the uncertainty of the automatic state, and avoids the inefficiency of simple manual remote control, so as to maximize the intelligence of the car. 


\section{Acknowledgements}

The work described in this paper was supported by General Project of Natural Science Foundation of College and University in Jiangsu Province(16KJB510002); Young teachers' Research and Development Foundation of Southeast University Chengxian College(y310001).

\section{References}

[1] Chen Dong. Research and implementation of intelligent car motion control system [M]. Nanjing: Southeast University, 2005

[2] Zhou Meili, Jiao Wenfang, Li Peng, et al. Control system design of intelligent vehicle based on electromagnetic field detection [J]. Foreign Electronic Measurement Technology, 2018.37 (3)

[3] Wang Liren, Zhu Yanyu, Huang Jie, et al. Research on control system of intelligent car with WiFi communication function. [J]. Wireless Interconnect Technology, 2014 (6)

[4] Yang Xiaofen, Li Zhenhua. Design of smart car for environmental monitoring based on Android platform [J]. Electronic World, 2016 (16)

[5] Chen Junru. Design of intelligent car system based on Android console[J]. Technology Innovation and Application, 2018 (8). 